\title{
Drug Discovery of $\beta$-Secretase Inhibitors Based on Quantum Chemical Interactions for the Treatment of Alzheimer's Disease
}

\author{
Yoshio Hamada* \\ Faculty of Pharmaceutical Sciences, Kobe Gakuin University, Minatojima, Chuo-ku, Japan
}

Received: September 01, 2014, Accepted: October 03, 2014, Published: October 17, 2014

*Corresponding author: Yoshio Hamada, Faculty of Pharmaceutical Sciences, Kobe Gakuin University, Minatojima, Chuo-ku, Kobe 650-8586, Japan, Tel: +81-78-974-1551; E-mail: pynden@gmail.com

\begin{abstract}
Alzheimer's disease, which is the most common cause of dementia, is characterized by progressive intellectual deterioration. According to the amyloid hypothesis, a transmembrane aspartic protease, $\beta$-secretase (BACE1), is a promising molecular target for development anti-Alzheimer drugs. This protease triggers amyloid $\beta$ $(A \beta)$ formation, which is involved in the development of Alzheimer's disease. Many research groups have shown various BACE1 inhibitors to be efficacious in the treatment of Alzheimer's disease. Given the fact that Swedish-mutant amyloid precursor protein (APP) is cleaved faster than wild-type APP, early BACE1 inhibitors were designed on the basis of the Swedish-mutant APP amino acid sequence and possess a substrate transition-state analogue. At an early stage of this research, we also reported a series of peptidic BACE1 inhibitors possessing a substrate transition-state analogue. Subsequently, nonpeptidic BACE1 inhibitors were designed using the peptidic inhibitors as lead compounds and an in-silico conformational structure-based drug design approach. These inhibitors showed potent inhibitory activities. Among these, some inhibitors exhibited effective BACE1 inhibition in cultured cells and significant reduction of $A \beta$ production in vivo. In the process, we found that a quantum chemical interaction between the inhibitors and an arginine side chain at the active site of BACE1 plays a crucial role in the mechanism of BACE1 inhibition. In fact, out of the publicly available X-ray crystal structures of BACE1-inhibitor complexes, most interact with the Arg235 side chain of BACE1 by a quantum chemical interaction. It is known that quantum chemical interactions involving an arginine side chain play an important role in molecular recognition in proteins. Hence, a series of potent BACE1 inhibitors that were optimized to focus on the quantum chemical interaction with the arginine side chain of BACE1 were designed. Furthermore, based on these quantum chemical interactions, we proposed a novel concept in medicinal science, the electron donor/acceptor bioisostere. Although the bioisostere is an important concept in the design of practical drugs, the classical bioisostere concept does not assume interactions by such quantum chemical effects. This review describes our peptidic BACE1 inhibitors produced using substrate-based design and non-peptidic BACE1 inhibitors produced using structure-based design approaches. Subsequently, the significance of quantum chemical interaction in medicinal chemistry is discussed, and potent small-sized BACE1 inhibitors and the first peptides with BACE1 inhibitory activities, which were designed based on quantum chemical interactions, are described.
\end{abstract}

Keywords: Alzheimer's Disease; Transition-State Analogue; Enzyme Inhibitor; Drug Design; Quantum Chemical Interaction

\section{Introduction}

Alois Alzheimer, a psychiatrist and neuropathologist, observed a 51-year-old female patient at Frankfurt Asylum in 1901. She showed strange behavioral symptoms, including loss of short-term memory. Dr. Alzheimer reported her symptoms following her death five years later. Unfortunately, because its cause was unclear until recently, there have been no treatment approaches for this neurodegenerative disorder, so called Alzheimer's disease $(\mathrm{AD})$, since that first report by Dr. Alzheimer over 100 years ago. According to a report by the World Health Organization (WHO), 35.5 million people were estimated to be living with dementia in 2010, and there are 7.7 million new cases of dementia each year [1]. Dementia causes tremendous damage and suffering to the patient and caregivers, namely the families and, because of the high medical care costs involved, it is an economic burden incurred by every country. If a treatment for $\mathrm{AD}$ is not developed in the future, there could be a negative impact on the economic growth of all countries.

A breakthrough was made through the genetic study of some familial $\mathrm{AD}$ (FAD) patients who were found to have mutations of the gene coding for amyloid precursor protein (APP) and presenilin. These mutations caused an increase in amyloid $\beta(A \beta)$ peptides, which are the main components of senile plaques in the brains of patients with AD. These results were seminal in promoting an amyloid hypothesis for AD pathology [2-5]. A $\beta$ s are produced from APP by two processing enzymes- $\beta$-secretase (BACE1; $\beta$-site APP-cleaving enzyme 1 ) and $\gamma$-secretase-that are potential molecular targets for the development of antiAD drugs [6-13]. On the basis of the amyloid hypothesis, various BACE1 inhibitors and $\gamma$-secretase inhibitors have been investigated as $\mathrm{AD}$ drug candidates by multiple research groups. This article supplements our earlier review of peptidic and non-peptidic BACE1 inhibitors with 
recent developments in this field [10-13]. It is known that quantum chemical interactions involving an arginine side chain play an important role for molecular recognition in proteins. We here in discuss the significance of quantum chemical interactions in drug discovery, and introduce the concept of small-sized BACE1 inhibitors, designed on the basis of quantum chemical interactions.

\section{AD Pathology and Design of BACE1 Inhibitors}

BACE1, a type I transmembrane aspartic protease, triggers $A \beta$ formation by cleaving APP at the $A \beta$ domain $\mathrm{N}$-terminus as shown in Figure 1. At the next stage in the amyloidogenic pathway of APP, $\gamma$-secretase cleaves at the C-terminus of the $A \beta$ domain, releasing the $A \beta$ that consists mainly of two molecular species, $A \beta_{42}$ and $A \beta_{40}$. $\mathrm{A} \beta_{42}$ shows greater neurotoxicity and aggregability than $\mathrm{A} \beta_{40}$ and appears to be a key biomolecular marker of $\mathrm{AD}$ pathogenesis. APP is a type I transmembrane protein with a large extracellular domain and has three isoforms resulting from the alternative splicing of its mRNA. Many mutations of the APP gene have been reported. The Swedish mutation, around the cleavage site of BACE1, induces the cleavage by BACE1, thereby increasing the levels of $A \beta_{42}$ and $A \beta_{40}$ in the brain of patients with this mutation. The mutations near the cleavage site of $\gamma$-secretase affect $A \beta$ formation or the $\mathrm{A} \beta_{42} / \mathrm{A} \beta_{40}$ formation ratio. Additionally, presenilin 1 and 2 , the catalytic components of the $\gamma$-secretase complex, are reported to be causative genes in early-onset FAD [14]. These mutations also appear to cause FAD, and these findings provide compelling evidence for the "amyloid hypothesis."

The aspartic protease BACE1 forms the transition state, which poses a tetrahedral structure with a substrate. Because the transition state has no covalent bond between a substrate and the catalytic residue at the active site of BACE1, unlike that in the case of serine and cysteine protease, its inhibitors can be logically designed using a computational approach. In the most widely accepted mechanism for an aspartic protease, a water molecule between the substrate and catalytic site is partially activated by an aspartic carboxylate anion and involved in a carbonyl carbon of the $\mathrm{P}_{1}$ amino acid residue in the substrate as shown in Figure 2. The carbonyl oxygen, in turn, captures a proton from another aspartic carboxylic acid group in the catalytic site, resulting in a tetrahedral intermediate, and then the substrate is cleaved into two peptide fragments. A transition-state analogue mimicking the tetrahedral transition state can strongly bind with the active site of aspartic protease, inhibiting the enzyme activity. Renin and HIV-1 protease are also classified as aspartic proteases, and their inhibitors with a transition state analogue were designed using computational approaches. Many BACE1 inhibitors were designed using similar techniques. Several transition-state analogues have been widely exploited for designing aspartic protease inhibitors such as statine, norstatine, hydroxyethylamine,

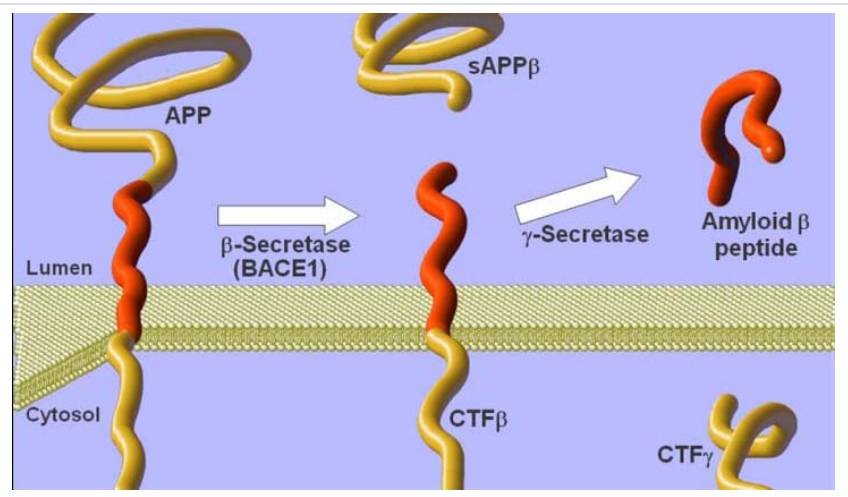

Figure 1: Processing pathway of amyloid precursor protein (APP). CTF = C-terminal fragment.

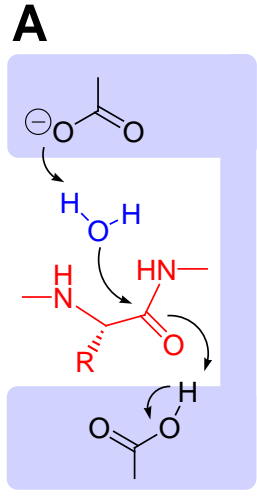

B

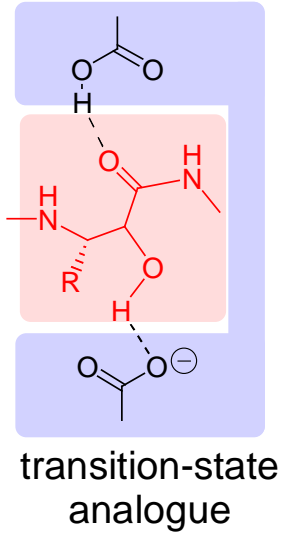

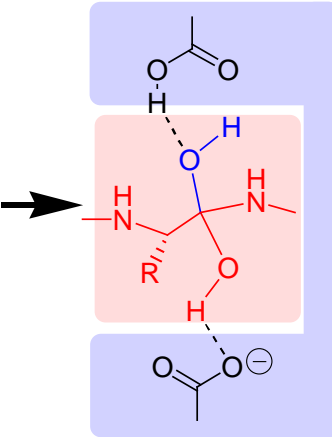

substrate transition state

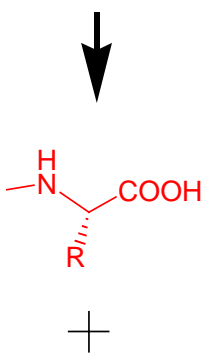

$\mathrm{H}_{2} \mathrm{~N}-$
Figure 2: (A) Peptide bond cleavage pathway via a substrate transition state by aspartic protease. (B) The norstatine-type substrate transitionstate analogue.

and hydroxyethylene units, and many BACE1 inhibitors possessing a transition-state analogue have been designed [11].

\section{Early Substrate-based BACE1 Inhibitors}

The Swedish-mutant APP gene, which contains a double mutation (K670N and M671L), has been found in patients with FAD. The Swedish-mutant APP sequence 
(EVNL*DAEF, Figure 3A) is cleaved faster than the wildtype APP sequence (EVKM*DAEF) by BACE1. Early BACE1 inhibitors possessed a transition-state analogue at the $\mathrm{P}_{1}$ position, and were designed from the Swedish-mutant APP sequence. In 1999, Sinha et al., working at Elan Pharmaceuticals, succeeded in purifying BACE1 from the human brain using a transition-state analogue, and cloned the enzyme [15]. The results provided the possibility for the use of substrate-based BACE1 inhibitors as AD drugs, leading to a boom in the development of BACE1 inhibitors. In 2000 and 2001, Ghosh and Tang et al. reported the potent inhibitors 0M99-2 $(\mathrm{Ki}=1.6 \mathrm{nM})$ and 0M00-3 $(\mathrm{Ki}=$ $0.3 \mathrm{nM}$ ) with a hydroxyethylene unit (corresponding to a dipeptide unit in this case) as a substrate transition-state analogue, and the first X-ray crystal structure (PDB ID: $1 \mathrm{FKN}$ ) of a complex between recombinant BACE1 and the inhibitor 0M99-2 [16-19]. We have reported on a series of peptidic BACE1 inhibitors possessing a norstatinetype transition-state analogue, phenyl norstatine [Pns: (2R,3S)-3-amino-2-hydroxy-4-phenylbutyric acid], at the $\mathrm{P}_{1}$ position as shown in Figure 4 [20-26]. These inhibitors have a bioisostere of a Glu residue at the $\mathrm{P}_{4}$ position and a substituted $\mathrm{N}$-phenylamide at the C-terminus. Of these inhibitors, 3 (KMI-429, IC $_{50}=3.9 \mathrm{nM}$ ) exhibited effective inhibition of BACE1 activity in cultured cells and significant reduction of $A \beta$ production in vivo (via direct administration into the hippocampi of APP transgenic and wild-type mice) $[20,21]$. Inhibitor $4\left(\mathrm{KMI}-684, \mathrm{IC}_{50}=1.2\right.$ $\mathrm{nM}$ ), in which two carboxylic acid residues of $\mathbf{3}$ at the $\mathrm{P}_{1}$ ' position were replaced with their bioisostere, a tetrazolyl group, showed the most potent BACE1 inhibitory activity [25]. Inhibitor 5 (KMI-574, $\left.\mathrm{IC}_{50}=5.6 \mathrm{nM}\right)$, possessing a 5 -fluoroortyl group in the $\mathrm{N}$-terminus residue, showed improved inhibition in cultured cells [24]. It is noteworthy that these inhibitors have a Leu residue at the $\mathrm{P}_{2}$ position. Interestingly, the compounds in which the Leu residues at the $\mathrm{P}_{2}$ position of these inhibitors were replaced with an Asn residue corresponding to the Swedish-mutant APP sequence, showed no BACE1 inhibitory activity.

\section{Non-peptidic BACE1 Inhibitors}

Many non-peptidic BACE1 inhibitors have been designed and synthesized using the peptidomimetic inhibitors as lead compounds and a structure-based drug design (SBDD) computational approach. Most non-peptidic inhibitors have an aromatic scaffold, such as an isophthalic group at the $\mathrm{P}_{2}$ position $[10,12]$. Probably, a plain molecule such as an aromatic ring appears to be preferred as a $\mathrm{P}_{2}$ moiety because of the narrow $\mathrm{S}_{2}$ pocket that is formed by the flap domain of BACE1.

We designed and synthesized non-peptidic BACE1 inhibitors, such as $\mathbf{6}$ and $\mathbf{7}$, with a norstatine-type transition-state analogue, allophenyl norstatine [Apns: (2S,3S)-3-amino-2-hydroxy-4-phenylbutyric acid], which is a diastereomer of Pns [27-31], as shown in Figure 5. Interestingly, they showed more potent inhibitory activities

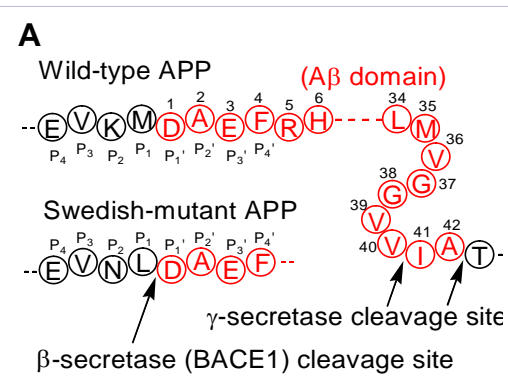

B

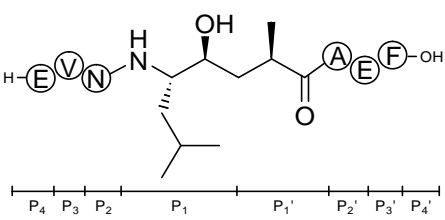

$1($ OM99-2) BACE $1 \mathrm{Ki}=1.6 \mathrm{nM}$

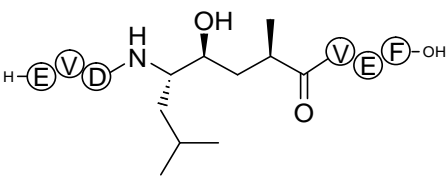

$2(\mathrm{OM} 00-3)$ BACE $1 \mathrm{Ki}=0.3 \mathrm{nM}$

Figure 3: (A) Cleavage sites of $\beta$ - and $\gamma$-secretase. (B) Peptidic $\beta$-secretase (BACE1) inhibitors by Ghosh et al.

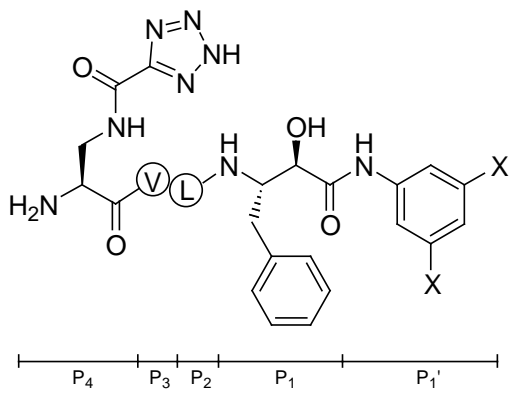

$3(\mathrm{X}=-\mathrm{COOH}, \mathrm{KMI}-429) \quad \mathrm{IC}_{50}=3.9 \mathrm{nM}$

$4\left(X=\underset{N=N^{\prime}}{N} N_{N H}, K M I-684\right) I_{50}=1.2 \mathrm{nM}$<smiles></smiles>

$\mathrm{C}_{\mathrm{A}}$ : cyclohexylalanine

5 (KMI-574) BACE $1 \mathrm{IC}_{50}=5.6 \mathrm{nM}$

Figure 4: Peptidic $\beta$-secretase (BACE1) inhibitors 
than the inhibitors possessing a Pns residue at the same position, contrary to the case of peptidic inhibitors 3-5. These inhibitors have a pyridinedicarboxylic or chelidonic scaffold at the $\mathrm{P}_{2}$ position and were designed from a virtual inhibitor with an isophthalic moiety at the $\mathrm{P}_{2}$ position using an in-silico conformational structure-based design approach [27,32]. Inhibitors 6 (KMI-1027, IC $\left.{ }_{50}=50 \mathrm{nM}\right)$ and 7 (KMI-1036, IC I0 $_{1}=96 \mathrm{nM}$ ) exhibited moderate BACE1 activities. The oxazolidine ring in inhibitors 6 and 7 fixes the direction of the phenyl ring at the $\mathrm{P}_{3}$ position, so the $\mathrm{P}_{3}$ phenyl ring can closely bind to the $\mathrm{S}_{3}$-subpocket of BACE1.

\section{Significance of Quantum Chemical Interactions in the Drug Discovery and Design of BACE1 Inhibitors}

In the first X-ray crystal structure (PDB ID: 1FKN) of a BACE1-inhibitor complex, it was found that the $\mathrm{P}_{2}$ Asn moiety, corresponding to the Swedish-mutant APP sequence, of the inhibitor 0M99-2 interacted with the Arg235 side chain at the active site of BACE1 by hydrogen bonding. Such hydrogen-bond interactions were thought to be important for binding between BACE1 and inhibitors. Subsequently, many BACE1 inhibitors possessing a hydrogen bond acceptor, such as an Asn residue at the $\mathrm{P}_{2}$ position, were reported. However, our potent inhibitors have a Leu residue at the $\mathrm{P}_{2}$ position, and compounds with an Asn residue at the same position showed no BACE1 inhibitory activity, as stated previously. We predicted that the interaction with the $S_{2}$ pocket might play a key role in the BACE1 inhibitory mechanism, because the Arg235 side chain in the $\mathrm{S}_{2}$ site is only found outside the opening of the active site formed by the flap domain of BACE1. Hence, we compared the publicly available X-ray crystal structures of BACE1-inhibitor complexes. Surprisingly, the guanidine group of BACE1-Arg235 in most of the crystal structures, except 1FKN, showed similar figures flopping over the $\mathrm{P}_{2}$ region of the inhibitors, and the nearest distances between the guanidino-plane of Arg235 and the $\mathrm{P}_{2}$ region show similar values of about $3 \AA$. This fact suggests that the guanidino-plane interacts with the $\mathrm{P}_{2}$ region by a weak quantum force such as stacking or $\sigma-\pi$ interaction [28]. The functional group found to interact with the Arg235 side chain in many of the crystal structures was the methyl group that was bound to the Arg235 side chain by a $\mathrm{CH}-\pi$ interaction. Subsequently, an $0-\pi$ interaction with an amide oxygen atom, $S-\pi$ interaction with a methionine sulfur atom, $\mathrm{CH}-\pi$ stacking with a pyridine proton, and $\pi-\pi$ stacking with an aromatic ring were observed. For instance, 3D views of the inhibitors in BACE1 (PDB ID: 4FSL and 2P83), which was reported by the researchers at Bristol-Myers Squibb (BMS) and Pfizer, were shown in Figure 6. These inhibitors interact with the Arg235 side chain by $\pi-\pi$ stacking and $0-\pi$ interaction, respectively. The Arg235 of the BACE1-OM99-2 complex (1FKN) assumed an exceptionally different pose to the other
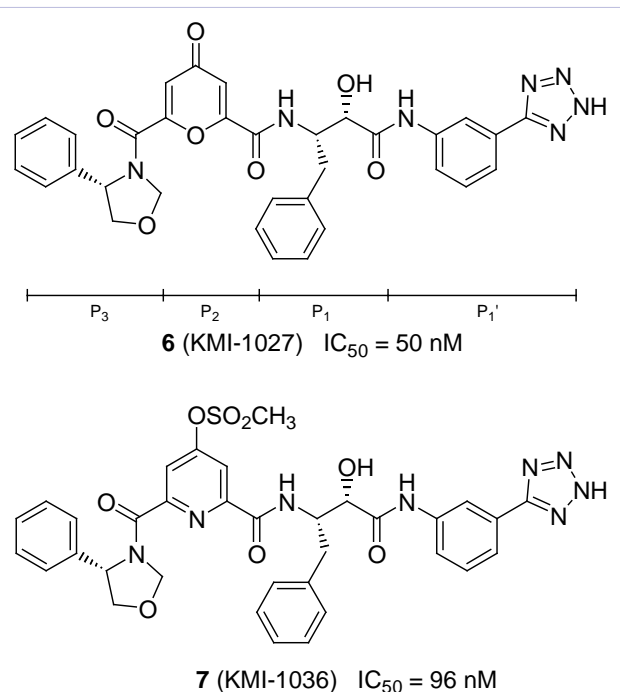

Figure 5: Non-peptidic $\beta$-secretase (BACE1) inhibitors.
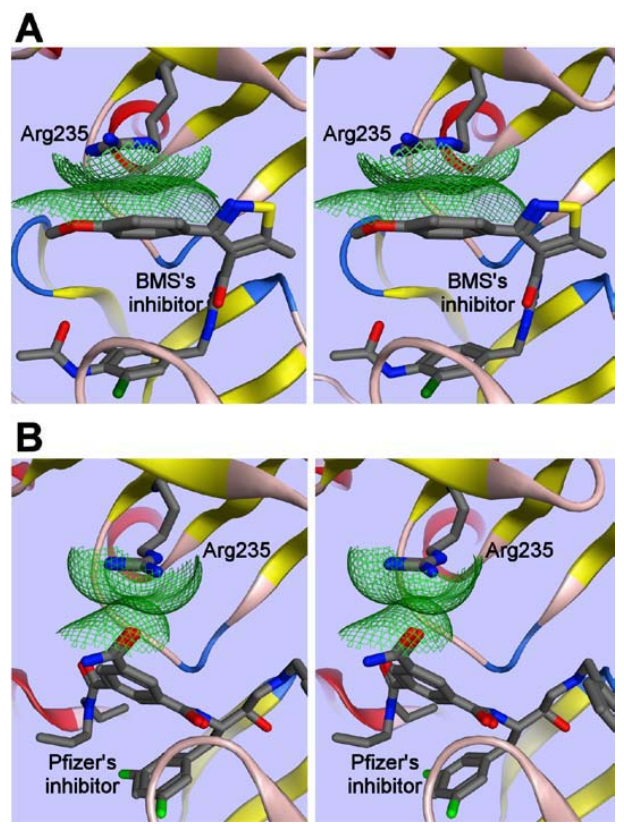

Figure 6: Quantum chemical interactions of BACE1-Arg235 side chain with inhibitors. (A) $\pi-\pi$ Stacking interaction of Arg235 side chain with the aromatic ring of BMS's inhibitor in the crystal structure (PDB ID: 4FSL). (B) 0- $\pi$ interaction of Arg235 side chain and the amide oxygen atom of Pfizer's inhibitor in the crystal structure (PDB ID: 2P83).

crystal structures. It was obvious from the fact that OM003 , developed by the same researchers, had no hydrogen bonding interaction with the Arg235 side chain in the crystal structure (PDB ID: 1M4H). The unique crystal structure of the BACE1-OM99-2 complex appears to be caused by the intramolecular hydrogen bonding between the $\mathrm{P}_{4}$-Glu and $\mathrm{P}_{2}$-Asn side chains of 0M99-2, because OM00-3, which is structurally similar to 0M99-2, has no intramolecular hydrogen bonding in the crystal structure. 
Computational approaches, such as docking simulation between a biomolecule and drugs, have greatly contributed to drug discovery research. However, most software for docking simulation adopt mechanism/ molecular dynamics (MM/MD) calculations based on classical Newtonian mechanics, and docking simulations using these calculations do not appear to estimate a weak quantum chemical interaction, such as stacking or $\sigma-\pi$ interaction between inhibitors and the Arg235 side chain of BACE1. The quantum chemical interactions that involve other aromatic amino acids, such as Phe, Tyr, and Trp, seem to be approximately optimized using several descriptors based on classical mechanics in the docking simulation software that is based on Newtonian mechanics. However, an arginine is recognized as a charged amino acid in this software, and the quantum chemical interactions involving an Arg side chain are unlikely to get a reasonable output.

It is well known that quantum chemical interactions involving a $\pi$-orbital of a guanidine group are found commonly in proteins and play a key role in molecular recognition by proteins. Crowley et al. surveyed cation- $\pi$ interactions in protein interfaces using the Protein Data Bank and the Protein Quaternary Structure server [33]. The cation- $\pi$ interactions were evaluated using a variant of the optimized potentials for liquid simulations (OPLS) force field. They found that approximately half of the proteinprotein complexes and one-third of the homodimers contained at least one intermolecular cation- $\pi$ pair. This finding indicates the significance of these interactions in molecular recognition, because the occurrence rate of cation- $\pi$ pairs in protein-protein interfaces is higher than that in homodimer interfaces, which are similar to the protein interior. Among them, the interactions between arginine and tyrosine were found to be the most abundant. Moreover, $53 \%$ of interactions between arginine and aromatic amino acids involved planar stacking, namely quantum chemical interactions, between the guanidine group and the aromatic ring. As an example to indicate the significance of quantum chemical interactions by an arginine side chain, Persson et al. reported the arginine switch mechanism for the molecular recognition of the CD46 receptor by an adenovirus [34]. A group of highly pathogenic species $B$ adenoviruses can infect a host using CD46 as a cellular receptor. The knob domain of the fiber protein on the capsid of an adenovirus mediates interactions with CD46. The knob domain of adenovirus type 11 (Ad11 knob) has two arginine residues (Arg279 and Arg280) at the recognition site for CD46. Since the Agr280 side chain interacts with the Arg279 side chain by a stacking interaction (on switch), the Arg280 side chain can stably bind to the Phe35 side chain of CD46 by a stacking interaction. These quantum chemical interactions play a critical role in the molecular recognition by the Ad11 knob of the CD46 receptor. Because the Arg279 of Ad11 is mutated into a Gln residue in the $\mathrm{Ad} 7$ and $\mathrm{Ad} 14 \mathrm{knob}$, the affinities between the Ad7/Ad14 knob and CD46 are known to be low. As observed above, quantum chemical interactions appear to be significant in medicinal science, but their treatment using the docking simulation software based on an MM/MD approach requires particular attention.

Non-peptidic BACE1 inhibitors 6 and 7 were designed using the docking simulation software Molecular Operating Environment (MOE, Chemical Computing Group Inc., Canada) on the basis of an MD calculation, and these inhibitors have a hydrogen-bond acceptor group based on the Swedish-mutant APP sequence. Of course, a quantum chemical interaction with the Arg235 side chain was not considered in the molecular design. Hence, we amended the design to focus on the quantum chemical interaction with the Arg235 side chain. An electron-rich halogen atom might interact with the electron-poor guanidine- $\pi$ orbital by Coulomb's force. If these interactions also involve chargetransfer interactions (CT) with directing effects, the effects are expected to be similar to stacking or $\sigma-\pi$ interactions with directing effects. Using the $a b$ initio molecular orbital approach, Imai et al. indicated that the calculated $\mathrm{Cl}-\pi$ interaction energy is slightly stronger than that of $\mathrm{CH}-\pi$ interaction and its energy is affected by $\pi$-electron density [35]. Inhibitors 8-10, with a halogen atom, were designed and these exhibited potent BACE1 inhibitory activities as shown in Figure 7 [28]. The most potent inhibitor, 9 (KMI$1303, \mathrm{IC}_{50}=9 \mathrm{nM}$ ), is available from Wako Pure Chemical Industries, Ltd (Japan), as a reagent for biological research. The fact that the introduction of a halogen atom at the $\mathrm{P}_{2}$ position in our compounds drastically improved BACE1 inhibitory activity appears to support our hypothesis, namely that the quantum chemical interactions between BACE1 and inhibitors, plays an important role in the mechanism of BACE1 inhibition; and the $\mathrm{P}_{2}$ part of a potent BACE1 inhibitor binds to the Arg235 side chain of BACE1, not by a hydrogen bonding interaction, but by a quantum chemical interaction.

\section{Electron Donor Bioisostere and Design of Peptides with BACE1 Inhibitory Activity}

Although most BACE1 inhibitors interact with the Arg235 side chain by quantum chemical interaction, it is known that the Swedish-mutant APP type substrate, possessing an Asn residue at the $\mathrm{P}_{2}$ position, elicits higher catalytic efficiency by BACE1 than the wild-type APP type substrate. The $K_{\mathrm{m}}$ values of both types of substrate are similar, suggesting that the affinities for both substrates against BACE1 are similar [36]. We considered that the hydrogen-bonding interaction between the Arg235 side chain and the $\mathrm{P}_{2}$-Asn side chain of the Swedish-mutant type substrates activate the "turnover" required for enzymatic catalysis, thereby improving the kcat value (ie. turnover number, a kinetic parameter). In contrast, such interaction appears to be unfavorable for BACE1 inhibition. In fact, most BACE1 inhibitors interact with the Arg235 side chain by a quantum chemical interaction. Generally, hydrogen- 


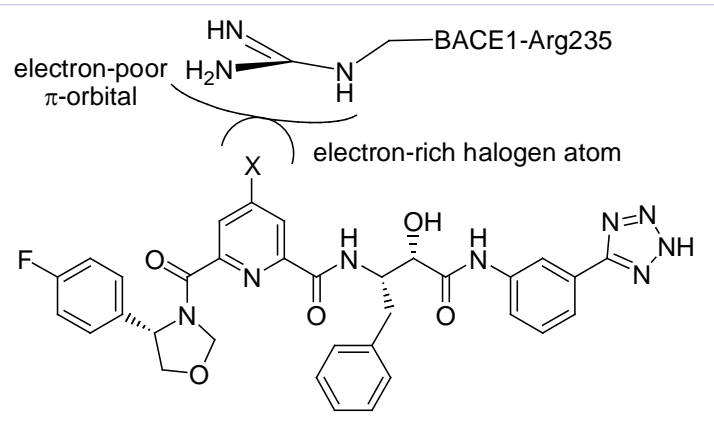

\begin{tabular}{rcc}
\hline Compound & $\mathrm{X}$ & BACE $1 \mathrm{IC}_{50}$ \\
\hline $\mathbf{8}$ (KMI-1283) & $\mathrm{Cl}$ & $13 \mathrm{nM}$ \\
$\mathbf{9}$ (KMI-1303) & $\mathrm{Br}$ & $9 \mathrm{nM}$ \\
$\mathbf{1 0}$ (KMI-1302) & $\mathrm{I}$ & $10 \mathrm{nM}$ \\
\hline
\end{tabular}

Figure 7: $\beta$-Secretase (BACE1) inhibitors based on quantum chemical interaction with the Arg235 side chain.

bonding interactions are obviously stronger than quantum chemical interactions, such as stacking or $\sigma-\pi$ interaction. However, we speculated that the guanidine group of Arg235 overlapped a $\mathrm{P}_{2}$ part of the inhibitors and a weak quantum chemical interaction with an inhibitor could fix it on the active site of BACE1 by inactivating the turnover of the BACE1 enzyme.

Introduction of the concept of "bioisostere" in the field of medicinal chemistry has revolutionized drug discovery process. Designing bioisosteres by applying molecular modeling approaches has resulted in improving the pharmacological properties of the 'active pharmaceutical ingredient'. Bioisosteres are functional or atomic groups that display similar physiochemical properties. To develop more practical drugs, compounds replaced with a bioisostere, which exhibit similar biological properties to the parent drugs, have been designed. However, the bioisostere of the ligand's $\mathrm{P}_{2}$ moiety according to the Swedish-mutant APP is an Asn bioisostere using a bioisostere concept that does not assume quantum chemical interactions, and inhibitors that can interact with the Arg235 side chain by a quantum chemical interaction could never be designed using such a classical concept. Hence, we proposed the new "electron-donor bioisostere," which can interact with an electron-poor $\pi$-orbital, such as the guanidine group of Arg235 by quantum chemical interactions.

Our potent peptidic BACE1 inhibitors such as $\mathbf{3 - 5}$, have a hydrophobic amino acid, such as Leu or Cha, at the $\mathrm{P}_{2}$ position, and their side chains appear to behave as an electron-donor bioisostere. Therefore, we proposed a novel hypothesis-If the $\mathrm{P}_{2}$ moiety of a substrate for BACE1 is replaced with an electron-donor bioisostere that can reduce the turnover of BACE1, would it turn into a BACE1 inhibitor? Hence, we designed and synthesized a series of peptides possessing a natural amino acid, Leu residue, as an electron-donor bioisostere as shown in Table 1 [37]. The octapeptide 11 exhibited weak inhibitory activities. Crucially, 11 is the first peptide that showed BACE1 inhibitory activity. The fact that a usual peptide without a transition-state analogue could inhibit BACE1 enzyme activity is encouragement for future research toward developing a gene-based therapy for Alzheimer's disease using DNA that codes for a peptide sequence with BACE1 inhibitory activity. As octapeptide 12, a D-amino acid residue, exhibited slightly higher inhibitory activity than 11, pentapeptides 13 and $\mathbf{1 4}$ possessing a D-amino acid were synthesized, and they replicated the inhibitory activity of 12. Previously, we reported that replacing the Glu residue at the $\mathrm{P}_{4}$-position of peptidic BACE1 inhibitors with its bioisostere, an $N^{\beta}$-(5-fluoroorotyl)-L2,3-diaminopropionic acid (5FO) residue, improved their BACE1 inhibitory activity [23]. Hence, a series of BACE1 inhibitors containing a 5-FO residue in the $\mathrm{P}_{4}$-amino acid residue were designed. Peptides 15-17 exhibited moderate BACE1 inhibitory activity. In contrast, peptides in which the $\mathrm{P}_{2}$-residue of these peptides was replaced with the $\mathrm{P}_{2}$ Asn residue corresponding to the Swedish-mutant APP sequence, exhibited no inhibitory activity, as predicted. Moreover, small-sized tripeptides 18-20 exhibiting BACE1 inhibitory activities were designed and synthesized using an in-silico conformational structure-based drug design approach [32]. Among them, tripeptide $19\left(\mathrm{IC}_{50}=50 \mathrm{nM}\right)$ exhibited potent BACE1 inhibitory activity.

\section{Conclusion}

We designed peptidic BACE1 inhibitors with a transition-state analogue using substrate-based design and non-peptidic BACE1 inhibitors using structure-based design approaches. Next, we found that a quantum chemical interaction between the inhibitors and an arginine side chain at the active site of BACE1 plays a crucial role in the mechanism of BACE1 inhibition, and designed a series of non-peptidic BACE1 inhibitors based on the quantum chemical interactions. Furthermore, we proposed the

\begin{tabular}{|c|c|c|c|}
\hline & Compound & $\begin{array}{c}\text { BACE } 1 \text { inhibition } \\
\% \text { at } 2 \mu \mathrm{M}\end{array}$ & $\mathrm{IC}_{50}(\mathrm{nM})$ \\
\hline 11 & 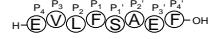 & 6 & - \\
\hline 12 & н-(B)(1)(L) (F) & 28 & - \\
\hline 13 & 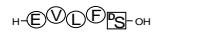 & 23 & - \\
\hline 14 & H-(E)(D)(D) & 29 & - \\
\hline 15 & 5F O-(D(L) $(\mathrm{P}$ (S)-он & 85 & - \\
\hline 16 & 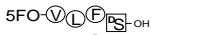 & 94 & 138 \\
\hline 17 & $5 \mathrm{FO}-($ (D)(B) $(\mathrm{P}-\mathrm{OH}$ & 90 & 184 \\
\hline 18 & $5 F O-\left(\mathbb{D}\left(D_{-1}^{-H} \times \mathbb{1}\right.\right.$ & 90 & - \\
\hline 19 & $5 F 0-(1)$ & 96 & 50 \\
\hline 20 & $5 \mathrm{FO}-(\nabla)(1)-N]$ & 91 & - \\
\hline
\end{tabular}

$$
\begin{aligned}
& \text { 可: D-serine } \\
& \text { [N : D-asparagine } \\
& \text { (CA): cyclohexylalanine } \\
& \text { 5F O: } N^{\beta} \text {-(5-fluoroorotyl)-L-2,3- } \\
& \text { diaminopropionic acid } \\
& \int_{0} \stackrel{1}{N}_{N H}
\end{aligned}
$$

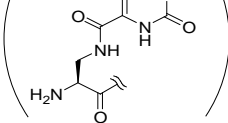

Table 1: $\beta$-Secretase (BACE1) inhibitor peptides with no transitionstate analogue. 
electron-donor bioisostere concept based on quantum chemical interactions, and designed the first peptides possessing BACE1 inhibitory activities. It is anticipated that such quantum chemical interactions will be applied to drug discovery research.

\section{Acknowledgement}

The studies were performed at Kobe Gakuin University and Kyoto Pharmaceutical University, and were supported in part by Grants-in-Aid for Scientific Research from MEXT (Ministry of Education, Culture, Sports, Science and Technology), Japan (KAKENHI No. 23590137 and No. 26460163)

\section{References}

1. World Health Organization. Dementia: A public health priority. Available from: http://whqlibdoc.who.int/ publications/2012/9789241564458_eng.pdf.

2. Selkoe DJ. Toward a comprehensivetheory for Alzheimer's disease. Hypothesis: Alzheimer's disease is caused by the cerebral accumulation and cytotoxicity of amyloid $\beta$-protein. Ann N Y Acad Sci. 2000; 924: 17-25.

3. Selkoe DJ. The deposition of amyloid proteins in the aging mammalian brain: implications for Alzheimer's disease. Ann Med. 1989; 21(2): 73 76.

4. Selkoe DJ. Translating cell biology into therapeutic advances in Alzheimer's disease. Nature. 1999; 399(6738 Suppl): A23-31.

5. Sinha S, Lieberburg I. Cellular mechanisms of $\beta$-amyloid production and secretion. Proc Natl Acad Sci U S A. 1999; 96(20): 11049-11053.

6. Vassar R, Bennett BD, Babu-Khan S, Kahn S, Mendiaz EA, Denis P, et al. $\beta$-Secretase cleavage of Alzheimer's amyloid precursor protein by the transmembrane aspartic protease BACE. Science. 1999; 286(5440): 735-741.

7. Hussain I, Powell D, Howlett DR, Tew DG, Meek TD, Chapman C, et al. Identification of a Novel Aspartic Protease (Asp 2) as $\beta$-Secretase. Mol Cell Neurosci. 1999; 14(6): 419-427.

8. Yan R, Bienkowski MJ, Shuck ME, Miao H, Tory MC, Pauley AM, et al Membrane-anchored aspartyl protease with Alzheimer's disease $\beta$-secretase activity. Nature. 1999; 402(6761): 533-537.

9. Sinha S, Anderson JP, Barbour R, Basi GS, Caccavello R, Davis D, et al. Purification and cloning of amyloid precursor protein $\beta$-secretase from human brain. Nature. 1999; 402(6761): 537-540.

10. Hamada H, Kiso Y. Recent progress in the drug discovery of nonpeptidic BACE1 inhibitors. Expert Opin Drug Discov. 2009; 4(4): 391416. doi: $10.1517 / 17460440902806377$.

11. Hamada $\mathrm{H}$, Kiso Y. The application of bioisosteres in drug design for novel drug discovery: focusing on acid protease inhibitors. Expert Opin Drug Discov. 2012; 7(10): 903-922.

12. Hamada H, Kiso Y. Advances in the identification of $\beta$-secretase inhibitors. Expert Opin Drug Discov. 2013; 8(6): 709-731. doi: 10.1517/17460441.2013.784267.

13. Nguyen JT, Hamada Y, Kimura T, Kiso Y. Design of potent aspartic protease inhibitors to treat various diseases. Arch Pharm (Weinheim). 2008; 341(9): 523-535. doi: 10.1002/ardp.200700267.

14. Citron M, Westaway D, Xia W, Carlson G, Diehl T, Levesque G, et al.
Mutant presenilins of Alzheimer's disease increase production of 42-residue amyloid -protein in both transfected cells and transgenic mice. Nat Med. 1997; 3(1): 67-72.

15. Sinha S, Anderson JP, Barbour R, Basi GS, Caccavello R, Davis D, et al. Purification and cloning of amyloid precursor protein $\beta$-secretase from human brain. Nature. 1999; 402(6761): 537-40.

16. Arun K. Ghosh , Dongwoo Shin , Debbie Downs, Gerald Koelsch , Xinli Lin , Jacques Ermolieff, et al. Design of potent inhibitors for human brain memapsin 2 ( $\beta$-secretase). J. Am. Chem. Soc. 2000; 122(14): 3522-3523. doi: $10.1021 / \mathrm{ja} 000300 \mathrm{~g}$.

17. Hong L, Koelsch G, Lin X, Wu S, Terzyan S, Ghosh AK, et al. Structure of the protease domain of memapsin 2 ( $\beta$-secretase) complexed with inhibitor. Science. 2000; 290(5489): 150-153.

18. Ghosh AK, Bilcer G, Harwood C, Kawahama R, Shin D, Hussain KA, et al. Structure-based design: potent inhibitors of human brain memapsin 2 ( $\beta$-secretase). J Med Chem. 2001; 44(18): 2865-2868.

19. Ghosh AK, Devasamudram T, Hong L, DeZutter C, Xu X, Weerasena V, et al. Structure-based design of cycloamide-urethane-derived novel inhibitors of human brain memapsin 2 ( $\beta$-secretase). Bioorg Med Chem Lett. 2005; 15(1): 15-20.

20. Kimura T, Shuto D, Hamada Y, Igawa N, Kasai S, Liu P, et al. Design and synthesis of highly active Alzheimer's $\beta$-secretase (BACE1) inhibitors, KMI-420 and KMI-429, with enhanced chemical stability. Bioorg Med Chem Lett. 2005; 15(1): 211-215.

21. Asai M, Hattori C, Iwata N, Saido TC, Sasagawa N, Szabó B, et al. The novel $\beta$-secretase inhibitor KMI-429 reduces amyloid beta peptide production in amyloid precursor protein transgenic and wild-type mice. J Neurochem. 2006; 96(2): 533-540.

22. Kimura T, Hamada Y, Stochaj M, Ikari H, Nagamine A, Abdel-Rahman $\mathrm{H}$, et al. Design and synthesis of potent $\beta$-secretase (BACE1) inhibitors with P1' carboxylic acid bioisostere. Bioorg Med Chem Lett. 2006; 16(9): 2380-2386.

23. Hamada $\mathrm{Y}$, Igawa $\mathrm{N}$, Ikari $\mathrm{H}$, Ziora $\mathrm{Z}$, Nguyen JT, Yamani $\mathrm{A}$, et al. $\beta$-Secretase inhibitors: modification at the $\mathrm{P}_{4}$ position and improvement of inhibitory activity in cultured cells. Bioorg Med Chem Lett. 2006; 16(16): 4354-4359.

24. Hamada Y, Abdel-Rahman H, Yamani A, Nguyen JT, Stochaj M, Hidaka K, et al. BACE1 inhibitors: optimization by replacing the $\mathrm{P}_{1}{ }^{\prime}$ residue with non-acidic moiety. Bioorg Med Chem Lett. 2008; 18(5): 1649-1653. doi: 10.1016/j.bmcl.2008.01.

25. Tagad HD, Hamada Y, Nguyen JT, Hamada T, Abdel-Rahman H, Yamani A, et al. Design of pentapeptidic BACE1 inhibitors with carboxylic acid bioisosteres at $\mathrm{P}_{1}^{\prime}$ and $\mathrm{P}_{4}$ positions. Bioorg Med Chem. 2010; 18(9): 3175-3186. doi: 10.1016/j.bmc.2010.03.032.

26. Tagad HD, Hamada Y, Nguyen JT, Hidaka K, Hamada T, Sohma $\mathrm{Y}$, et al. Structure-guided design and synthesis of $\mathrm{P}_{1}^{\prime}$ position 1-phenylcycloalkylamine-derived pentapeptidic BACE1 inhibitors. Bioorg Med Chem. 2011; 19(17): 5238-5246. doi: 10.1016/j. bmc.2011.07.002.

27. Hamada Y, Ohta H, Miyamoto N, Yamaguchi R, Yamani A, Hidaka K, et al. Novel non-peptidic and small-sized BACE1 inhibitors. Bioorg Med Chem Lett. 2008; 18(5): 1643-1647. doi: 10.1016/j.bmcl.2008.01.056.

28. Hamada Y, Ohta H, Miyamoto N, Sarma D, Hamada T, Nakanishi T, et al. Significance of interaction of BACE1-Arg235 with its ligands and design of BACE1 inhibitors with $\mathrm{P}_{2}$ pyridine scaffold. Bioorg Med Chem Lett. 2009; 19(9): 2435-2439. doi: 10.1016/j.bmcl.2009.03.049. 
29. Hamada Y, Nakanishi T, Suzuki K, Yamaguchi R, Hamada T, Hidaka K, et al. Novel BACE1 inhibitors possessing a 5-nitroisophthalic scaffold at the $\mathrm{P}_{2}$ position. Bioorg Med Chem Lett. 2012; 22(14): 4640-4644. doi: 10.1016/j.bmcl.2012.05.089.

30. Suzuki K, Hamada Y, Nguyen JT, Kiso Y. Novel BACE1 inhibitors with a non-acidic heterocycle at the $\mathrm{P}_{1}{ }^{\prime}$ position. Bioorg Med Chem. 2013; 21(21): 6665-6673. doi: 10.1016/j.bmc.2013.08.016.

31. Hamada Y, Suzuki K, Nakanishi T, Sarma D, Ohta H, Yamaguchi R, et al. Structure-activity relationship study of BACE1 inhibitors possessing a chelidonic or 2,6-pyridinedicarboxylic scaffold at the $\mathrm{P}_{2}$ position. Bioorg Med Chem Lett. 2014; 24(2): 618-623. doi: 10.1016/j. bmcl.2013.12.007.

32. Hamada Y, Tagad HD, Nishimura Y, Ishiura S, Kiso Y. Tripeptidic BACE1 inhibitors devised by in-silico conformational structure-based design Bioorg Med Chem Lett. 2012; 22(2): 1130-1135. doi: 10.1016/j. bmcl.2011.11.102.
33. Crowley PB, Golovin A. Cation- $\pi$ interactions in protein-protein interfaces. Proteins. 2005; 59(2): 231-239.

34. Persson BD, Müller S, Reiter DM, Schmitt BB, Marttila M, Sumowski CV, et al. An arginine switch in the species B Adenovirus knob determines high-affinity engagement of cellular receptor CD46. J Virol. 2009; 83(2): 673-686. doi: 10.1128/JVI.01967-08.

35. Imai YN, Inoue Y, Nakanishi I, Kitaura $\mathrm{K} . \mathrm{Cl}-\pi$ interactions in proteinligand complexes Protein Sci. 2008; 17(7): 1129-1137. doi: 10.1110/ ps.033910.107.

36. Grüninger-Leitch F, Schlatter D, Küng E, Nelböck P, Döbeli H. Substrate and Inhibitor Profile of BACE ( $\beta$-Secretase) and Comparison with Other Mammalian Aspartic Proteases. J Biol Chem. 2002; 277(7): 4687-4693.

37. Hamada Y, Ishiura S, Kiso Y. BACE1 Inhibitor Peptides: Can an Infinitely Small $k_{\text {cat }}$ Value Turn the Substrate of an Enzyme into Its Inhibitor? ACS Med. Chem. Lett. 2013; 3(3): 193-197. doi : 10.1021/ml2002373. 